\title{
Technical Considerations in Brain Amyloid PET Imaging with ${ }^{18}$ F-Florbetapir
}

\author{
LisaAnn Trembath, CNMT, MSM, CCRA, FSNMMI-TS, Maureen Newell, CNMT, RT(N), and Michael D. Devous, Sr., PhD
}

Avid Radiopharmaceuticals, Inc., Philadelphia, Pennsyvania

CE credit: For CE credit, you can access the test for this article, as well as additional JNMT CE tests, online at https://www.snmmilearningcenter.org. Complete the test online no later than September 2018. Your online test will be scored immediately. You may make 3 attempts to pass the test and must answer $80 \%$ of the questions correctly to receive $1.0 \mathrm{CEH}$ (Continuing Education Hour) credit. SNMMI members will have their CEH credit added to their VOICE transcript automatically; nonmembers will be able to print out a CE certificate upon successfully completing the test. The online test is free to SNMMI members; nonmembers must pay $\$ 15.00$ by credit card when logging onto the website to take the test.

Technical factors play a critical role in the production of bestquality amyloid PET images for interpretation. This article provides specific instructions and general technical information about PET brain scanning of $\beta$-amyloid neuritic plaques. The focus of tracer-specific information will be on ${ }^{18} \mathrm{~F}$-florbetapir (indications, contraindications, dosing, administration, uptake time, scanning time, acquisition, processing, biodistribution, radiation dose, adverse events, and display). General scanning information relevant to all amyloid-imaging agents will be also be presented (e.g., mechanism of uptake, safe handling, positioning, prevention of patient motion, processing, and artifacts).

Key Words: florbetapir; PET; brain imaging; amyloid; technical

J Nucl Med Technol 2015; 43:175-184

DOI: 10.2967/jnmt.115.156679

$\beta_{\text {of } \beta}$ -amyloid neuritic plaques are extracellular deposits of $\beta$-amyloid protein mixed with branches of dying nerve cells found in the cortical gray matter of patients with Alzheimer disease. These toxic proteins, along with the intracellular neurofibrillary tangles of tau protein, are implicated in the disease process, although the exact sequence and timing of their development is not completely understood (1). Until recently, clinicians could study the regional distribution of these proteins only in the laboratory, using specimens of brain tissue obtained at autopsy or biopsy. With the advent of PET amyloid-imaging agents, a new era in the study of neurodegenerative disorders is under way in which the in vivo density and distribution of amyloid protein in the brains of living subjects can be estimated (2).

The first successful PET tracer for amyloid imaging in humans was ${ }^{11} \mathrm{C}$-Pittsburgh compound $\mathrm{B}$. The PET signal

\footnotetext{
Received Mar. 4, 2015; revision accepted Jun. 16, 2015.

For correspondence or reprints contact: LisaAnn Trembath, CNMT, MSM, CCRA, FSNMTS, Avid Radiopharmaceuticals, Inc., 3711 Market St., Philadelphia, PA 19104.

E-mail: Trembath@avidrp.com

Published online Aug. 13, 2015.

COPYRIGHT (c) 2015 by the Society of Nuclear Medicine and Molecular Imaging, Inc.
}

from this tracer correlates with the location and density of $\beta$-amyloid neuritic plaques as assessed in vitro (3). Because of the physical limitations in the production of ${ }^{11} \mathrm{C}$ and its short half-life (20.3 min), investigators have sought ways to image $\beta$-amyloid plaques with ${ }^{18} \mathrm{~F}$-labeled compounds that can more easily be translated from academic research centers into widespread clinical use (2). Three ${ }^{18} \mathrm{~F}$-labeled amyloid PET radiopharmaceuticals have been approved by the U.S. Food and Drug Administration and the European Medicines Agency: ${ }^{18}$ F-florbetapir (Amyvid; Eli Lilly \& Co.) $(4,5)$, flutemetamol (Vizamyl; GE Healthcare) $(6,7)$, and florbetaben (Neuraceq; Piramal Imaging) $(8,9)$.

\section{INDICATIONS AND CONTRAINDICATIONS}

PET amyloid agents bind to amyloid neuritic plaque in cortical gray matter, scans of which provide an in vivo estimation of plaque density. All 3 approved agents share a similar indication statement. The product indication as cited in the U.S. package insert (USPI) for ${ }^{18} \mathrm{~F}$-florbetapir is as follows. ${ }^{18} \mathrm{~F}$-florbetapir is a...

radioactive diagnostic agent for Positron Emission Tomography (PET) imaging of the brain to estimate $\beta$-amyloid neuritic plaque density in adult patients with cognitive impairment who are being evaluated for Alzheimer's Disease (AD) and other causes of cognitive decline (4).

Specific indication language in the European Union Summary of Product Characteristics (SPC) is consistent with that of the USPI (5). The implications of a negative and positive scan are described in the ${ }^{18} \mathrm{~F}$-florbetapir USPI as follows:

\footnotetext{
A negative $\left[{ }^{18} \mathrm{~F}\right.$-florbetapir] scan indicates sparse to no neuritic plaques, and is inconsistent with a neuropathological diagnosis of $\mathrm{AD}$ at the time of image acquisition; a negative scan result reduces the likelihood that a patient's cognitive impairment is due to $\mathrm{AD}$.
}

A positive $\left[{ }^{18} \mathrm{~F}\right.$-florbetapir] scan indicates moderate to frequent amyloid neuritic plaques; neuropathological examination has shown this amount of amyloid neuritic plaque is present in patients with $\mathrm{AD}$, but may also be present in patients with other types of neurologic conditions as well as older people with normal cognition (4).

An amyloid brain scan is not a stand-alone test, and a positive result does not establish a diagnosis of Alzheimer 
disease; rather, it is part of a diagnostic work-up by a clinician and should be considered an adjunct to neuropsychologic testing and other elements of a patient evaluation. An amyloid scan informs the clinician about the density of amyloid plaque in the brain, which is one factor involved in the diagnostic evaluation of cognitive impairment (10). Per the ${ }^{18} \mathrm{~F}$-florbetapir USPI, the "Safety and effectiveness of $\left[{ }^{18} \mathrm{~F}\right.$-florbetapir] has not been established for: predicting development of dementia or other neurologic condition; [or for] monitoring responses to therapies." ${ }^{18} \mathrm{~F}$-florbetapir imaging is an estimate of plaque density at the time of imaging only; it does not predict future development of $\beta$-amyloid neuritic plaque $(4,5)$.

${ }^{18} \mathrm{~F}$-florbetapir is not indicated for pediatric patients. Women of childbearing years should follow standard radiation precautions and inform their clinician if they could possibly be pregnant or if they are breastfeeding. As with any radiopharmaceutical, patients who are breastfeeding should be advised to use alternate infant nutrition sources after administration. Injection of ${ }^{18} \mathrm{~F}$-florbetapir (or any radiopharmaceutical) contributes to cumulative radiation dose $(4,5)$.

There is no contraindication listed in the USPI for ${ }^{18} \mathrm{~F}$ florbetapir (4). The SPC cites hypersensitivity to the active component or inactive ingredients as a contraindication for use (5).

Errors may occur in interpretation with any of the amyloidimaging agents. Motion artifacts, atrophy, image noise, suboptimal positioning, oversmoothing, and surgery- or radiationinduced changes can affect the accuracy of interpretation. Anatomic correlation with CT or MR imaging is useful in determining the location and severity of atrophy and other brain disease such as stroke or ventriculomegaly that could interfere with interpretation (4-9).

\section{ADVERSE REACTIONS}

Adverse reactions that occurred during ${ }^{18} \mathrm{~F}$-florbetapir clinical trials were documented and submitted to regulatory agencies as part of the application for marketing approval. In any clinical trial, not only are adverse event data collected from specific patient groups that may or may not match the population typically exposed to a new drug after approval, but the total number of subjects exposed to an investigational drug is much smaller than the number with eventual access to the drug in routine clinical practice. For these reasons, the rate and total number of adverse events should not be directly extrapolated to clinical practice or compared between products (11). Because ${ }^{18} \mathrm{~F}$-florbetapir clinical studies did not include a placebo group, it is not possible to statistically distinguish adverse events occurring more frequently in patients given drug versus patients undergoing similar procedures without drug treatment.

In clinical trials of ${ }^{18} \mathrm{~F}$-florbetapir $(n=555)$, there were no serious adverse reactions; reported reactions were predominantly mild to moderate. The USPI lists the most frequent adverse reactions reported by more than one subject in clinical trials: headache $(1.8 \%)$; musculoskeletal pain, increased blood pressure, and nausea $(0.7 \%)$; and fatigue and injection site reaction $(0.5 \%)$. The following adverse reactions were reported to have occurred in $0.4 \%$ of subjects $(2 / 555)$ : anxiety, back pain, claustrophobia, dizziness, feeling cold, insomnia, and neck pain (4). The SPC categorizes adverse reactions slightly differently from the USPI. Headache is listed as occurring with "common" frequency, meaning that its range of occurrence was $\geq 1$ in 100 but $<1$ in 10 . The adverse reactions that were listed as "uncommon" ( $\geq 1$ in 1,000 but $<1$ in 100) are dysgeusia (distortion of the sense of taste), flushing, nausea, pruritus, urticaria, and infusion site rash (5). The difference in the descriptions between the USPI and the SPC are a matter of how the regulatory agencies categorize and report adverse reaction data and does not indicate a difference in the raw data between the two regions.

\section{MECHANISM OF ACTION AND BIODISTRIBUTION}

Amyloid-imaging agents such as ${ }^{18} \mathrm{~F}$-florbetapir diffuse across the blood-brain barrier and produce a radioactive signal that is detectable throughout the brain. Washout of unbound ligand ensues, leading to decreasing tracer in the brain, followed by differential retention between areas that contain $\beta$-amyloid aggregates (which are a major component of neuritic plaques) and areas that do not. This differential in tracer uptake is the basis for determining whether the scan is positive or negative for amyloid (4-9). Beyond this general similarity, however, each ${ }^{18} \mathrm{~F}$ amyloid-imaging agent has distinct chemistry, clearance, and biodistribution profiles.

In the case of ${ }^{18} \mathrm{~F}$-florbetapir, brain uptake maximizes within several minutes of injection and then clears rapidly from the circulation during the first $30 \mathrm{~min}$ after injection. Clearance slows significantly after $30 \mathrm{~min}$, and by $40-50 \mathrm{~min}$ the activity remains in a steady state until $90 \mathrm{~min}$ after injection (12). ${ }^{18} \mathrm{~F}$ florbetapir is eliminated primarily by clearance through the liver and excretion into the gallbladder and intestines $(4,5)$.

In a study of healthy subjects, ${ }^{18} \mathrm{~F}$-florbetapir was distributed throughout the body, and the amount of injected ${ }^{18} \mathrm{~F}$ in the blood was less than $5 \%$ by 20 min after injection and less than $2 \%$ by $45 \mathrm{~min}$. Some accumulation and excretion was also observed in the urinary bladder. Activity in the urine was due to polar metabolites rather than the parent compound. The liver was visualized within 4 min after injection. Residual ${ }^{18} \mathrm{~F}$ in the circulation during the 30 - to 90 -min imaging window consisted primarily of polar metabolites $(4,5)$.

The highest level of ${ }^{18} \mathrm{~F}$-florbetapir uptake in the normal brain is in regions comprising mostly white matter. The reason for this nonspecific uptake in white matter has not been definitively established. One hypothesis is that, because of slower cerebral blood flow in white matter than in gray matter, ${ }^{18}$ F-florbetapir clears more slowly from white matter (5).

${ }^{18}$ F-florbetapir uptake has been identified in nonbrain structures such as scalp, salivary glands, muscles, and bone. It is unknown whether such uptake is due to blood accumulation of ${ }^{18} \mathrm{~F}$-florbetapir or of any of its radioactive metabolites (5).

\section{RADIATION DOSIMETRY}

Amyloid PET radiopharmaceuticals, used as directed in the product labeling, result in an effective whole-body dose of radiation that is similar to that from an ${ }^{18} \mathrm{~F}-\mathrm{FDG}$ PET 
scan (7 mSv for $370 \mathrm{MBq}$ [10 mCi] of ${ }^{18}$ F-FDG) $(4-9,13)$. The dosimetry for each amyloid tracer is based on the specific biodistribution and kinetics of the tracer. The target organ (i.e., the organ with the highest radiation dose) for ${ }^{18} \mathrm{~F}$-florbetapir is the gallbladder wall (Table 1). The effective whole-body dose for $370 \mathrm{MBq}(10 \mathrm{mCi})$ of ${ }^{18} \mathrm{~F}$-florbetapir is $7 \mathrm{mSv}(19 \mu \mathrm{Sv} /$ $\mathrm{MBq})(4,5)$.

The radiation dose from the $\mathrm{CT}$ portion of the PET/CT scan is operator- and scanner-dependent and may range to up to $2.2 \mathrm{mSv}$ or more for a diagnostic brain scan but may be much less for a low-dose (attenuation correction) scan (13). The decision to use diagnostic-dose versus low-dose CT is based on the specific clinical scenario and each institution's standards and, as such, is beyond the scope of this article.

\section{PATIENT PREPARATION}

Because amyloid-imaging agents are not susceptible to changes in metabolism, it is not necessary to monitor blood glucose or require patients to fast before injection. Unlike ${ }^{18} \mathrm{~F}-\mathrm{FDG}$, the conditions of injection and uptake are not restricted. Patients can be in a lighted room with ambient noise and can talk, watch television, eat, drink, walk, and interact with others (in compliance with institution-specific radiation safety policies) during the uptake time after injection.

TABLE 1

18F-Florbetapir-Estimated Absorbed Radiation Dose (4)

\begin{tabular}{|c|c|}
\hline Organ or tissue & $\mu \mathrm{Gy} / \mathrm{MBq}$ \\
\hline Adrenal & 14 \\
\hline \multicolumn{2}{|l|}{ Bone } \\
\hline Osteogenic cells & 28 \\
\hline Red marrow & 14 \\
\hline Brain & 10 \\
\hline Breasts & 6 \\
\hline Gallbladder wall & 143 \\
\hline \multicolumn{2}{|l|}{ Gastrointestinal tract } \\
\hline Lower large intestine wall & 28 \\
\hline Small intestine & 66 \\
\hline Stomach wall & 12 \\
\hline Upper intestine wall & 74 \\
\hline Heart wall & 13 \\
\hline Kidneys & 14 \\
\hline Liver & 64 \\
\hline Lungs & 9 \\
\hline Muscle & 9 \\
\hline Ovaries & 18 \\
\hline Pancreas & 14 \\
\hline Skin & 6 \\
\hline Spleen & 9 \\
\hline Testes & 7 \\
\hline Thymus & 7 \\
\hline Thyroid & 7 \\
\hline Urinary bladder wall & 27 \\
\hline Uterus & 16 \\
\hline Total body & 12 \\
\hline Effective dose* & $19^{*}$ \\
\hline${ }^{*} \mu \mathrm{Sv} / \mathrm{MBq}$ & \\
\hline
\end{tabular}

There are no medication restrictions for ${ }^{18} \mathrm{~F}$-florbetapir imaging. Patients can continue taking their dementia medications and other medications as prescribed. Drug-to-drug interactions were not studied in clinical trials of ${ }^{18} \mathrm{~F}$-florbetapir. However, subjects in clinical trials were not told to stop taking their medications, including those commonly used to treat symptoms of cognitive impairment (e.g., donepezil, galantamine, and memantine). A comparison of data between subjects who were on versus not on dementia medications showed no difference in quantitative values $(4,5)$.

The SPC for ${ }^{18} \mathrm{~F}$-florbetapir suggests that patients should avoid any close contact with young children and pregnant women for the $24 \mathrm{~h}$ after the injection. In addition, the following specific warnings are cited in the SPC (5):

The content of sodium is greater than $1 \mathrm{mmol}$ (up to $37 \mathrm{mg}$ per dose). This should be taken into account in patients on a low sodium diet.

This medicinal product contains 10 vol\% ethanol (alcohol), i.e. up to $790 \mathrm{mg}$ per dose, equivalent to $20 \mathrm{~mL}$ beer or $8 \mathrm{~mL}$ wine per dose. This amount may be harmful for those suffering from alcoholism, and should be taken into account in pregnant and breast-feeding women and high-risk groups such as patients with liver disease or epilepsy.

Metal or attenuating materials in the field of view for any PET brain scan, ${ }^{18}$ F-FDG or amyloid, cause CT artifacts, which can in turn cause errors in attenuation correction and PET image artifacts (14-16). The technologist should ensure that all metal or attenuating materials above the shoulders have been removed to avoid CT artifacts. Examples of such materials are glasses, hearing aids, removable dentures, and hair clips.

\section{DOSING AND ADMINISTRATION}

As with all radiopharmaceuticals for injection, ${ }^{18} \mathrm{~F}$ florbetapir should be inspected for particulate matter; if particulates are seen, the dose should not be injected. ${ }^{18} \mathrm{~F}$ florbetapir is a clear and colorless solution. The $\mathrm{pH}$ range is 5.5-7.5. ${ }^{18} \mathrm{~F}$-florbetapir should be stored at room temperature $\left(25^{\circ} \mathrm{C}, 77^{\circ} \mathrm{F}\right)$, with permitted excursions to $15^{\circ} \mathrm{C}-30^{\circ} \mathrm{C}$ $\left(59^{\circ} \mathrm{F}-86^{\circ} \mathrm{F}\right)$. The product does not contain a preservative (4).

Aseptic injection technique and appropriate radiation (high-energy) shielding, such as a tungsten syringe shield with a lead glass window, should be used when injecting PET agents. Waterproof gloves should be worn. Per the principle of "as low as reasonably achievable," technologists should apply time, distance, and shielding techniques to minimize the radiation dose to themselves and their colleagues. Unused product should be disposed of in a safe manner in compliance with applicable regulations (4-9).

${ }^{18}$ F-labeled amyloid-imaging agents are neutral and lipophilic_-characteristics that allow these agents to cross the blood-brain barrier (17-19). Moderate lipophilicity also means that these agents tend to adhere to plastic tubing and syringes. Technologists should use short catheters and as little extension tubing as possible to minimize adsorption of radiotracer, which could result in a decrease in injected dose. The ${ }^{18} \mathrm{~F}$-florbetapir product label recommends use of 
a short indwelling catheter of approximately $4 \mathrm{~cm}$ (1.5 in) or less to minimize adsorption to the catheter $(4,5)$.

The recommended dose of ${ }^{18} \mathrm{~F}$-florbetapir is $370 \mathrm{MBq}$ $(10 \mathrm{mCi})$ in a maximum volume of $10 \mathrm{~mL}$ administered via intravenous bolus, followed by a saline flush.

In the United States, ${ }^{18} \mathrm{~F}$-florbetapir is supplied to manufacturing sites in $10-, 30-$, or $50-\mathrm{mL}$ vials containing $10,10-30$, or $10-50 \mathrm{~mL}$, respectively, at a strength of 500$1,900 \mathrm{MBq}(13.5-51 \mathrm{mCi}) / \mathrm{mL}$ at the end of synthesis. Unit doses of ${ }^{18} \mathrm{~F}$-florbetapir are drawn by a commercial radiopharmacy and delivered to imaging sites in 6-mL polypropylene or high-density polyethylene syringes that, in addition to being made of material that results in less adsorption, have no rubber stoppers that could contribute to adsorption and a resulting loss of radioactivity. These syringes are slightly shorter than the standard 5-mL syringes but fit into a standard 5-mL PET syringe shield. If a 3-way stopcock is used, both ports should be rinsed thoroughly with saline to minimize residual activity. ${ }^{18} \mathrm{~F}$-florbetapir should not be diluted in the unit dose form (e.g., saline should not be drawn into the syringe to dilute the dose or increase the volume) (4).

In the European Union, ${ }^{18} \mathrm{~F}$-florbetapir is supplied to imaging sites in a multidose vial with concentrations of either $800 \mathrm{MBq} / \mathrm{mL}$ (range, $800-12,000 \mathrm{MBq}$ ) or $1,900 \mathrm{MBq} / \mathrm{mL}$ (range, 1,900-28,500 MBq) at the time of calibration. The volume administered to the patient should be minimally $1 \mathrm{~mL}$ and maximally $10 \mathrm{~mL}$. The ${ }^{18} \mathrm{~F}$-florbetapir SPC states that only polypropylene or high-density polyethylene syringes should be used when a dose is drawn from the vial. ${ }^{18} \mathrm{~F}$ florbetapir from the vial may be diluted aseptically with sodium chloride solution for injection to a maximum of 1:5. Diluted product must be used within $4 \mathrm{~h}$ of dilution (5). Automated injector and dispenser systems are not recommended for ${ }^{18} \mathrm{~F}$-florbetapir because of incompatibility with the internal silicon tubing used in many of the automated devices (Avid Radiopharmaceuticals, unpublished data, 2010).

The maximum mass dose of ${ }^{18} \mathrm{~F}$-florbetapir (i.e., amount of nonradioactive precursor compound) is $50 \mu \mathrm{g}(4,5)$. Unit doses or vials delivered from the radiopharmacy will indicate a product expiration date and time. A dose that is drawn before this date should contain the correct mass dose-volume proportion. However, an expired vial may require a larger volume of solution, which could potentially lead to injection of a mass dose larger than prescribed. The shelf-life for ${ }^{18} \mathrm{~F}-$ florbetapir $800 \mathrm{MBq} / \mathrm{mL}$ is $7.5 \mathrm{~h}$ from the time of calibration. For ${ }^{18} \mathrm{~F}$-florbetapir $1,900 \mathrm{MBq} / \mathrm{mL}$, the shelf-life is $10 \mathrm{~h}$. If ${ }^{18} \mathrm{~F}$-florbetapir is diluted (as is allowed in the European Union), it must be used within $4 \mathrm{~h}$ of dilution (5). Attention to the expiration date of radiopharmaceutical doses is universally good nuclear medicine practice for technologists.

\section{POSITIONING THE PATIENT}

Proper positioning of the patient contributes to ease of interpretation, minimizes potential artifacts due to motion, and promotes patient comfort. Many of the same position- ing aids and concepts used to optimize ${ }^{18} \mathrm{~F}$-FDG PET brain imaging can be applied to amyloid brain imaging.

One helpful way to standardize the description of positioning in the head holder is to use the terminology of airplane pilots in describing the orientation of an airplane in flight. Pitch is used when the nose of the plane moves up or down and is an apt description of the movement of a patient nodding his or her head. Yaw is used for a simple turn, such as when a patient turns to look to the right or left, or if the patient's nose is not at exactly the 12-o'clock position as seen in the axial view. Roll is the term used if one wing of an airplane dips lower than the other; the same can be said about one ear being lower than the other as if dipping toward the shoulder (20).

Patients should be positioned supine with their head in a nonattenuating head holder. The brain should be in the center of the scanner field of view to leverage maximum sensitivity of the detector, and the field should include the entire cerebellum. The head should be centered on the $y$-axis with the nose pointing straight up, with no yaw or roll, using a laser positioning system if available. The neck should be in a relaxed position, and the chin pitched slightly forward so that an imaginary line drawn from the external canthus of the eye to the center of the auditory meatus is perpendicular to the scanning table. If the patient cannot hold his or her head in this canthomeatal positioning, imaging can be performed with the neck completely relaxed and the images subsequently reoriented; however, with small-field-of-view scanners it may be difficult to contain the entire cerebellum within the field of view.

Patient positioning plays a key role in allowing the interpreter to follow the standardized method of interpretation and in producing images similar to those in the manufacturer-developed interpreter training program. For example, if the head is pitched too far forward or backward, images become more complicated to interpret because the interpreter must visualize anatomy in an atypical orientation while scrolling through slices. A scan that demonstrates left-to-right yaw or ear-to-shoulder roll can be more difficult to interpret because bilateral activity in a slice may appear asymmetric (Fig. 1A). Although reorientation software can reposition processed data such as pitch or yaw in 1 or 2 dimensions, not all software can adjust the image properly when there is a combination of noncentered positions (e.g., yaw combined with roll). Ensuring that the patient's head is centered in all 3 axes is an important way that the technologist can support a standardized appearance and subsequent interpretation of ${ }^{18} \mathrm{~F}$-florbetapir scans.

Motion is the most common cause of artifacts in PET brain imaging and is often preventable by using standard head-imaging techniques. Patient motion during a PET scan can reduce accuracy by causing image blurring within and between frames, misregistration between the CT and PET data, or scatter estimation and attenuation correction errors due to transmission and emission misalignment (21). Amyloid PET imaging is also vulnerable to blurring artifacts, which can potentially decrease accuracy (10). In a multicenter 


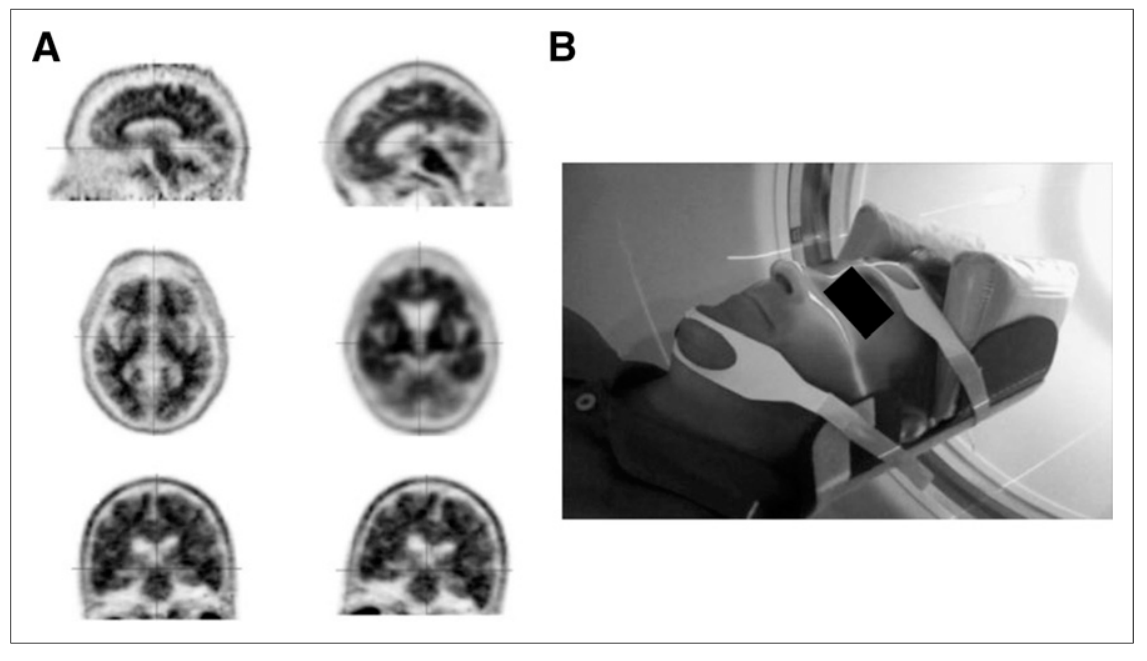

FIGURE 1. (A) Top row of images demonstrates proper canthomeatal positioning on left and head pitched too far forward on right. In middle row are corresponding axial projections, with standard appearance on left and nonstandard appearance on right, with frontal lobes prominent and occipital lobes just becoming visible. Bottom two images demonstrate proper coronal positioning (left), and right image shows prominent roll to left. (Courtesy of Avid Radiopharmaceuticals.) (B) Volunteer properly positioned in head holder (GE Healthcare) using laser system, cushions, chin strap, and forehead strap. (Courtesy of Desert Advanced [RadNet Imaging Centers], Palm Springs, California.) study conducted by the Japanese Alzheimer's Disease Neuroimaging Initiative, subjects with Alzheimer disease showed a higher level of motion during ${ }^{18} \mathrm{~F}-\mathrm{FDG}$ PET brain imaging than did those with mild cognitive impairment or no cognitive impairment (22). Green et al. studied head motion in cognitively normal cooperative volunteers by positioning them in a head holder and observing the amount and quality of motion on PET scans both with and without the use of thermoplastic masks. The authors observed that motion occurred in multiple planes, and cumulative motion increased with time even in cognitively normal volunteers who were made comfortable with appropriate positioning support. Motion was less frequent and severe with a mask than without any restraints (23). Beyer et al. studied the impact of patient support structures on head movement in a simulated PET/CT environment and concluded that motion and misregistration artifacts occurred most often when only the neck was supported and least often when the head holder was fitted with a custom foam insert. Because not all imaging centers have access to a curved head holder as in that study, the authors recommend using other types of cushioning along the sides of the head such as vacuum-lock bags or bean-filled bags. They also noted that a common source of downward motion is shoulder and neck relaxation during the acquisition (24).

To minimize the potential for motion artifacts in amyloid imaging, the patient's head should be secured firmly in the head holder with tape or a self-adhering wrap (e.g., Coban; $3 \mathrm{M})$ across the forehead, as well as a chin strap or tape. If there is still room for the patient's head to yaw right or left or for the ear to roll toward the shoulder, the technologist should use foam wedges, towels, or vacuum bags to secure the head (Fig. 1B). Some technologists find it useful to lie on the table and allow a colleague to position their head in the head holder using the chin and forehead strap. This experiment usually helps them better understand what the patient experiences and how to minimize potential artifacts.

The technologist should ask patients to relax their shoulders before the acquisition begins. One simple technique is to ask patients to squeeze their shoulders tight up to the ears for a few seconds and then let the shoulder muscles relax completely. Patients should be monitored closely for motion and reminded frequently to remain still. In some patients with dementia, a family member or caregiver may be the person best suited to encourage the patient to remain still during the acquisition. After completing the acquisition, the technologist should immediately review the images for motion or any other artifacts and rescan the patient if feasible. In those rare cases in which the patient cannot remain still during the entire scan, sedating antianxiety medication has been used. This decision, however, should be made by the primary care physician while taking into account the patient's symptoms and concurrent medications and may not be appropriate for all patients.

One way to mitigate potential motion artifacts is by acquiring scan data in smaller increments as a dynamic protocol. In this case, instead of one static data collection, data are collected in several shorter sequences that add up to the total acquisition time required. This technique has been used successfully for amyloid imaging by the Alzheimer disease Neuroimaging Initiative in the United States and Japan. For example, dynamic frames of 5 min each can be summed to form 10-, 15-, or 20-min datasets, with the option of dropping a frame that contains significant motion. The resulting scan would have fewer counts but would not be blurred by motion artifacts. Alternatively, if movement between frames is apparent, the affected frames can be spatially aligned and summed to produce a study with minimal motion effect (22). Figure 2 shows an example of artifacts caused by motion between two 5-min frames in a dynamic acquisition.

Ultimately the best way to avoid motion artifacts is to obtain a raw dataset without motion. If the above techniques are not successful or if, on reviewing the data immediately after the scan, the technologist sees evidence of patient motion, the acquisition may need to be repeated. The decision on whether to repeat the acquisition must be based on whether the patient can tolerate an additional examination and be able to remain still more successfully during a second scan than during the first. In the case of 
FIGURE 2. Ten-minute dynamic PET acquisition, with frame 1 at top and frame 2 at bottom. Frame 2 is representative of subject motion, most notably yaw and roll. Dashed line indicates degree of motion between frames. Arrows indicate image blurring. (Courtesy of Avid Radiopharmaceuticals.)

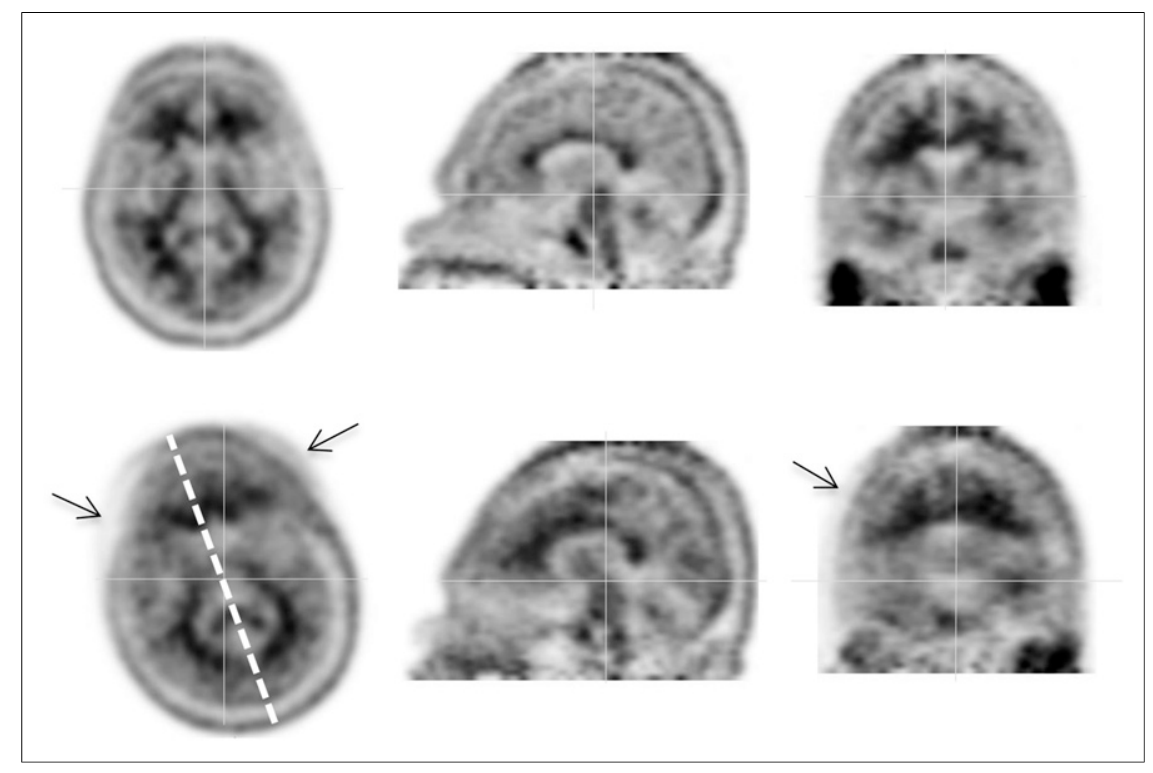

${ }^{18}$ F-florbetapir, a scan can be repeated as late as $80 \mathrm{~min}$ after injection. Acquisitions later than that are not recommended.

Enhancement of patient comfort is important and can lead to an examination that is less stressful for patient, caregiver, and technologist alike. It is also logical to presume that a patient who is warm and comfortable is less likely to move during the acquisition. Many camera rooms are cold, and the technologist can offer a warm blanket to keep the patient from shivering or becoming restless because of feeling chilled. The technologist should apply common techniques to promote patient comfort, such as pillows or a foam wedge under the knees for lumbar comfort and an abdominal strap to hold the arms in place so that arm muscles and shoulders can relax. These techniques also help to prevent movement in lower extremities or torso, which can result in head motion (25). Some patients may find it more comfortable to have their feet taped together to allow their legs and knees to relax into position. It is generally recommended that a sleeping patient should not be awakened during the acquisition because being startled may result in more motion than if the patient remains asleep. The Alzheimer's Disease Neuroimaging Initiative recommends that patients be offered a panic button or at least reassurance that someone is watching or able to hear them at all times. The patient should continually be reminded to remain still (26).

\section{SCANNING PARAMETERS}

Incubation, or uptake time, after injection is based on tracer uptake and selective retention in areas that retain the tracer versus those that do not. The recommended window for starting the ${ }^{18} \mathrm{~F}$-florbetapir PET scan is 30-50 min after injection. In most ${ }^{18} \mathrm{~F}$-florbetapir PET clinical studies, images were obtained at approximately $50 \mathrm{~min}$ after injection, but scans may start as early as $30 \mathrm{~min}$ and as late as $80 \mathrm{~min}$ after injection of the dose. Images should be acquired for $10 \mathrm{~min}$ in either static or dynamic mode $(4,5)$.
Three-dimensional acquisition is recommended for amyloid imaging because of the increased sensitivity relative to 2-dimensional imaging. Attenuation correction and appropriate scatter and energy corrections should be applied as with any PET scan (27). In general, it is recommended that amyloid images be acquired with only a single bed position. However, in the case of an extremely large head or small field of view, two bed positions may be required.

CT parameters for attenuation correction are not specific for amyloid PET versus ${ }^{18} \mathrm{~F}-\mathrm{FDG}$ PET, and technologists can apply a standard CT protocol as would be applied for ${ }^{18}$ F-FDG PET brain imaging in their institution. As previously mentioned, the decision to use diagnostic-dose versus low-dose CT is based on the specific clinical scenario and each institution's standards and, as such, is beyond the scope of this article. Generally, however, a low-dose CT protocol is adequate for attenuation correction and anatomic correlation for amyloid PET/CT.

\section{PROCESSING PARAMETERS}

Iterative reconstruction or ordered-subsets expectation maximization is generally recommended for ${ }^{18} \mathrm{~F}$-florbetapir imaging, although filtered backprojection is acceptable. The optimal number of iterations and subsets is usually less than that applied to ${ }^{18}$ F-FDG data because amyloid images tend to be lower-count studies than a typical $370 \mathrm{MBq}(10 \mathrm{mCi}){ }^{18} \mathrm{~F}-\mathrm{FDG}$ scan. The count rate and total counts for amyloid images are not predictable because the density of information depends on the amount of amyloid plaque. Scans with abnormal findings - a higher density of $\beta$-amyloid neuritic plaque-have a higher count rate than scans with negative findings-little to no plaque. Therefore, it is critical that images be acquired for the correct amount of time as directed in the product label rather than for a specific number of total counts. The 


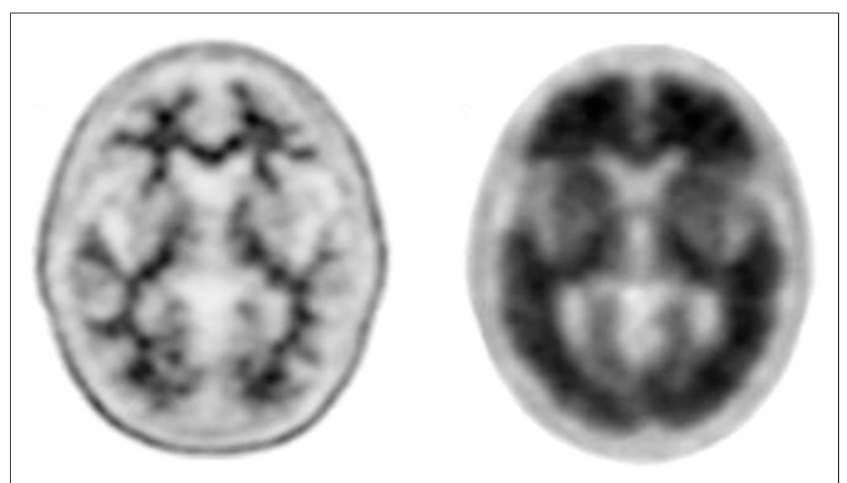

FIGURE 3. Representative amyloid-negative (left) and amyloid-positive (right) transaxial slices from ${ }^{18} \mathrm{~F}$-florbetapir PET scans. (Courtesy of Avid Radiopharmaceuticals.)

following range of iterations/subsets has been successfully used for ${ }^{18} \mathrm{~F}$-florbetapir imaging: 4/14, 4/15, 4/16, 3/20, and 3/21 (Avid Radiopharmaceuticals, unpublished data, 2014).

A $128 \times 128$ matrix was used in clinical trials of ${ }^{18} \mathrm{~F}$ florbetapir. Late-model PET/CT scanners can produce excellent images using a finer matrix, such as 256 or 336. Zoom should be set at 2 for most scanners. For systems that require a defined diagnostic field of view, $35 \mathrm{~cm}$ is recommended. A postprocessing gaussian filter with a full width at half maximum (FWHM) of 3-5 mm should generally be applied. As with any PET data, lowering the FWHM will apply more smoothing, and increasing the FWHM will result in sharper but noisier images. Similarly, a finer matrix may increase spatial resolution but at the cost of additional noise.

Time-of-flight and point-spread-function algorithms have not been extensively studied with amyloid imaging and were not included in clinical trials of ${ }^{18} \mathrm{~F}$-florbetapir. It is not known whether these high-resolution recovery techniques are beneficial for non-hotspot imaging in the brain, such as amyloid plaque imaging. Technologists should apply these techniques with caution and discuss the implications of the algorithms with the camera manufacturers. More research in this area is needed.

\section{DISPLAY AND INTERPRETATION}

Display and interpretation methods vary significantly among the ${ }^{18}$ F-labeled agents. Display techniques (i.e., color vs. gray scale) have been validated in clinical trials for the different tracers, and the label-recommended interpretation methods therefore differ. Technologists should be aware of the recommended method of display for the amyloid-imaging agent being used. Although each of the ${ }^{18} \mathrm{~F}$ agents has been demonstrated to reliably measure the density of $\beta$-amyloid neuritic plaque, understanding how the images are interpreted is important to using them successfully in clinical practice. Amyloid images should be interpreted only by those who have completed a training program germane to the specific radiopharmaceutical being used. For all 3 amyloid-imaging compounds, the objective of interpretation is to estimate neuritic plaque density, not to make a clinical diagnosis of Alzheimer disease $(4,5)$.

Amyloid images should be interpreted independently of the patient's clinical history and features. In many departments the PET technologist routinely records a basic patient history that may also include the referring clinician's notes on the patient's symptoms or cognitive status. This practice is not recommended for amyloid brain imaging, however, because interpreters are instructed to interpret the amyloid image in a manner that is masked to clinical status. Clinical trials of all 3 amyloid agents were performed with the interpreter masked to patient symptoms and history. Image interpretation that includes consideration of the patient's symptoms or clinical history may lead to errors (4-9). However, once the image results have been interpreted by the imaging physician, the clinician should interpret the results in the context of the patient's history

${ }^{18}$ F-florbetapir images should be displayed and interpreted by using a linear inverse gray scale (black on white), with the maximum intensity of the scale set to the maximum intensity of brain pixels (rather than by setting the maximum intensity to nonbrain structures such as salivary glands). All transaxial slices should be reviewed, starting at the cerebellum and moving upward through the brain. Sagittal and coronal images should be reviewed periodically as needed to better define radioactivity uptake and ensure that the entire brain is displayed. ${ }^{18} \mathrm{~F}$-florbetapir images are designated as positive or negative for amyloid by comparing activity in cortical gray matter with activity in adjacent white matter. This determination is made only in the cerebral cortex; the signal in the cerebellum does not contribute to scan interpretation (e.g., a positive scan may show retained cerebellar gray-white contrast even when the cortical gray-white contrast is lost). This method of interpretation is referred to as a binary method, meaning that the answer is only 1 of 2 choices: amyloidpositive or amyloid-negative. The text below, from the

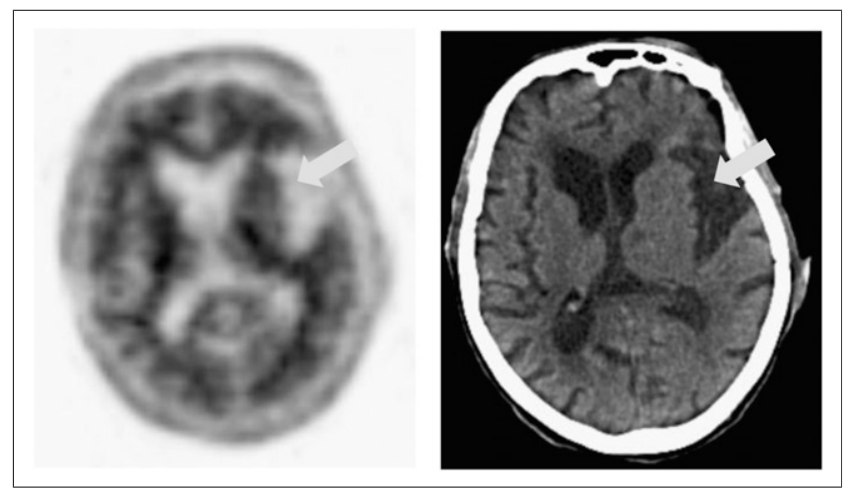

FIGURE 4. ${ }^{18} \mathrm{~F}$-florbetapir PET slice (left) and corresponding CT slice (right), with arrows indicating location of atrophy. (Courtesy of Avid Radiopharmaceuticals.) 
FIGURE 5. ${ }^{18} \mathrm{~F}$-florbetapir images with poor resolution due to dose infiltration (A), imaging beyond recommended time window (B), inadequate smoothing $(2 \mathrm{~mm}$ FWHM) (C), and scanner issue during acquisition (D). (Courtesy of Avid Radiopharmaceuticals.)

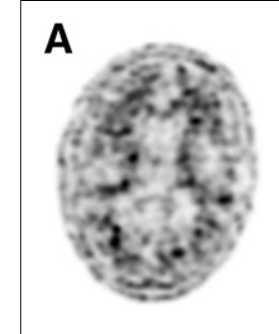

B

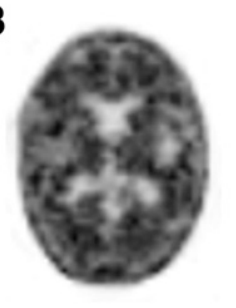

C

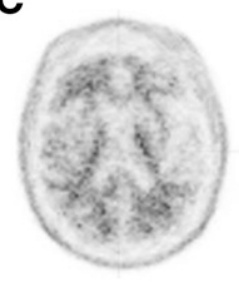

D

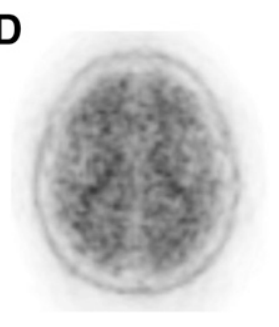

${ }^{18} \mathrm{~F}$-florbetapir USPI, defines a positive scan and a negative scan (4).

Negative $\left[{ }^{18} \mathrm{~F}\right.$-florbetapir] scans show more radioactivity in white matter than gray matter, creating clear gray-white contrast.

Positive scans show cortical areas with reduction or loss of the normally distinct gray-white contrast. These scans have one or more areas with increased cortical gray matter signal which results in reduced (or absent) gray-white contrast. Specifically, a positive scan will have either:

(a) Two or more brain areas (each larger than a single cortical gyrus) in which there is reduced or absent gray-white contrast. This is the most common appearance of a positive scan.

or

(b) One or more areas in which gray matter radioactivity is less intense and clearly exceeds radioactivity in adjacent white matter.

Figure 3 provides an example of an amyloid-negative scan and an amyloid-positive scan.

Quantitative analysis of amyloid images is widely used for research studies (10) but currently is not generally incorporated into clinical use. All three ${ }^{18} \mathrm{~F}$ amyloid-imaging tracers have been approved for visual interpretation, and as described in the product labels, quantitation software is not necessary to perform amyloid imaging (4-9). The use of quantitation as an adjunct to visual interpretation is, how- ever, currently undergoing evaluation. There are several commercially available quantitation software packages for amyloid imaging in the United States and the European Union.

\section{ARTIFACTS, PITFALLS, AND NORMAL VARIANTS}

Whether from normal aging or neurodegenerative disease, atrophy of the cerebral cortex decreases the thickness of the gray matter, making it more difficult to distinguish between gray and white matter, particularly in the frontal, temporal, and parietal lobes. Correlation with anatomic imaging such as CT can more clearly reveal the gray matter and clarify the relationship between amyloid tracer uptake and gray matter anatomy (4-9). Figure 4 shows an example of atrophy seen on an ${ }^{18} \mathrm{~F}$-florbetapir PET/CT image.

Other concomitant factors such as stroke, ventriculomegaly, surgical changes, post-radiation therapy changes, and implants can confound interpretation, but interpretation is often possible if gray-white contrast can be assessed in the nonaffected areas. Motion artifacts can cause blurring and misregistration between the CT and PET images, resulting in errors in attenuation correction and less accurate interpretation.

Excessively noisy images can be caused by low information density due to dose infiltration, scanning beyond the recommended imaging time, or undersmoothing of
FIGURE 6. (A) Normal salivary gland uptake on ${ }^{18} \mathrm{~F}$-florbetapir PET image. (B) Nonspecific bony uptake in skull and clivus bone confirmed by CT. (C) Nonspecific uptake in clivus bone. (D) Nonspecific uptake in skull. (Courtesy of Avid Radiopharmaceuticals.)

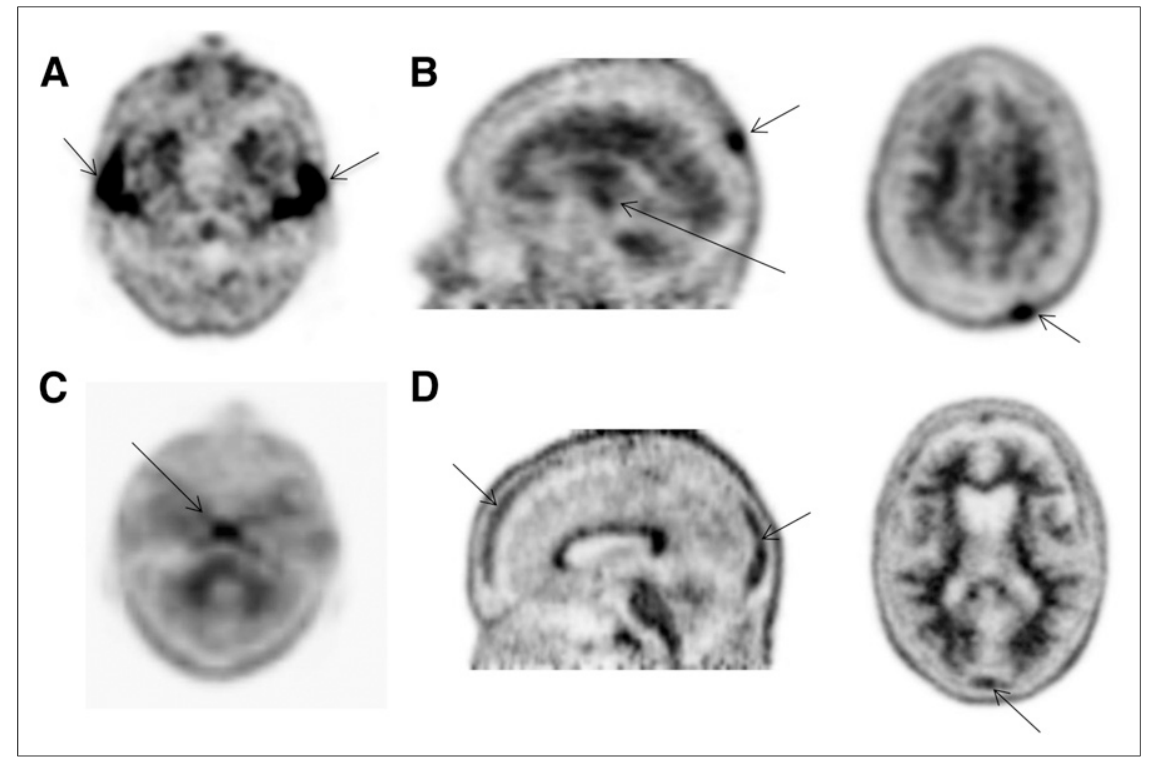




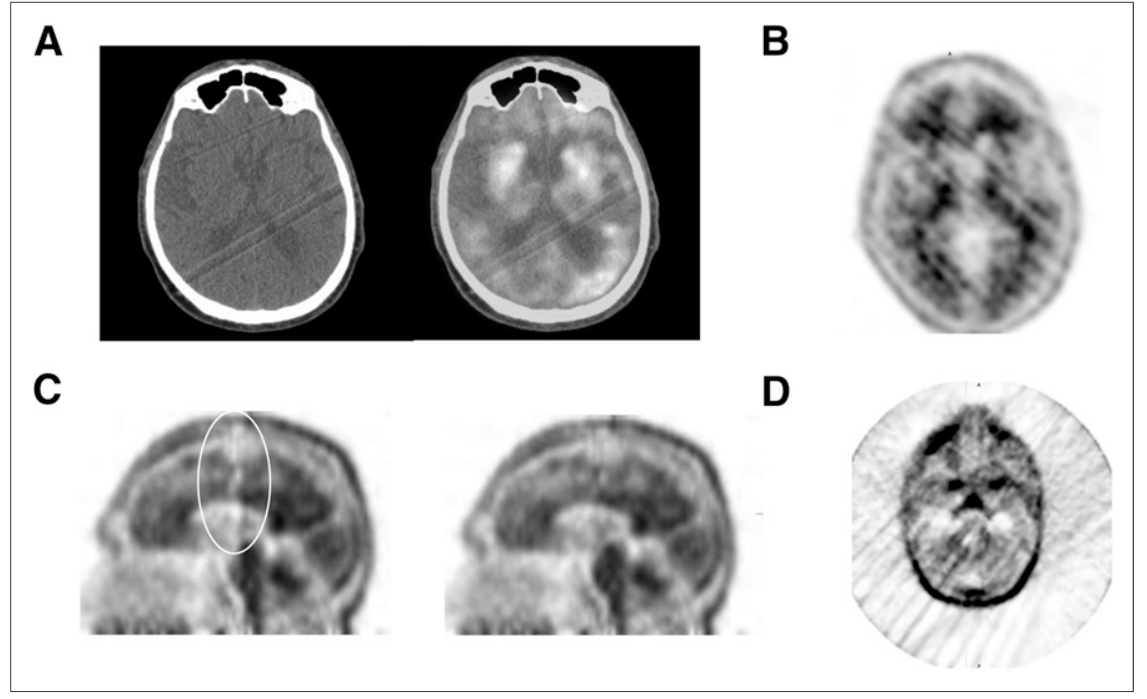

FIGURE 7. (A) Streak artifact on CT image (left) and PET/CT image (right) due to incomplete warm-up of CT tube. (B) Image resulting from detector block failure. (C) Image resulting from calibration error due to out-of-date normalization (left), and reprocessed image with new normalization (right). (D) Out-of-date normalization seen as streaks on nonattenuation-corrected images. (Courtesy of Avid Radiopharmaceuticals.) reconstructed images. Figure 5 provides examples of images that may not be interpretable because of excessive image noise.

Metal within the field of view can result in CT artifacts that have been shown to cause attenuation correction artifacts in ${ }^{18} \mathrm{~F}$-FDG PET images (14-16).

Interpreters and technologists should understand normal patterns of nonspecific uptake, such as uptake in the salivary glands and bony structures. Figure 6 provides examples of normal variants of nonspecific uptake found on ${ }^{18} \mathrm{~F}$-florbetapir images.

Like any PET procedure, amyloid PET is vulnerable to artifacts caused by absence of or inadequate quality control of instrumentation. The camera manufacturers' specifications for routine quality control procedures, corrections, and normalizations should be followed, including specifications for the CT portion of PET/CT scanners. Figure 7 shows examples of amyloid image artifacts resulting from inadequate quality control.

\section{CONCLUSION}

Amyloid PET imaging allows in vivo assessment of the amyloid status of a patient with cognitive impairment. The currently available PET radiopharmaceuticals for amyloid imaging bind to $\beta$-amyloid neuritic plaques in the brain and provide an accurate and reliable estimation of plaque density. No specific patient preparation is required beyond removing metal from the field of view. Positioning of the head and prevention of patient motion are critical to obtaining high-quality images. Up-to-date corrections and instrumentation quality control should be part of any amyloid-imaging program.

Each amyloid-imaging agent has unique dosing and administration instructions and a recommended scanning start time and duration, radiation dosimetry, biodistribution, and method of display and interpretation. Technologists should refer to the product label for tracer-specific details. In the case of ${ }^{18} \mathrm{~F}$-florbetapir, the dose is $370 \mathrm{MBq}$ $(10 \mathrm{mCi})$, injected intravenously followed by a saline flush.
Ten-minute scans should begin 30-50 min after injection. ${ }^{18}$ F-florbetapir images should be displayed with a linear inverse gray scale, with all 3 planes available to the interpreter.

Technical factors can either degrade or optimize amyloid image quality, which in turn has a direct effect on accurate interpretation of amyloid status. Technologists who know the characteristics of radiopharmaceuticals for amyloid PET, how to prepare and optimally position the patient, the correct scanning parameters, the recommended reconstruction and display methods, and how to avoid image artifacts will be better able to provide health care that makes a difference in the lives of those with Alzheimer disease and other causes of cognitive impairment.

\section{DISCLOSURE}

The authors are employees of Avid Radiopharmaceuticals, Inc., a wholly-owned subsidiary of Eli Lilly and Company. No other potential conflict of interest relevant to this article was reported.

\section{REFERENCES}

1. Querfurth HW, LaFerla FM. Mechanisms of disease: Alzheimer's disease. $N$ Engl J Med. 2010;362:329-344.

2. Mathis CA, Mason NS, Lopresti BJ, Klunk WE. Development of positron emission tomography $\beta$-amyloid plaque imaging agents. Semin Nucl Med. 2012; 42:423-432.

3. Klunk WE, Engler H, Nordbert A, et al. Imaging brain amyloid in Alzheimer's disease with Pittsburgh compound-B. Ann Neurol. 2004;55:306319.

4. Amyvid ${ }^{\text {TM }}$ (florbetapir F 18 injection) for intravenous use. Eli Lilly and Company website. pi.lilly.com/us/amyvid-uspi.pdf. Published 2012. Revised December 2013. Accessed July 8, 2015.

5. EPAR summary for the public: Amyvid florbetapir $\left({ }^{18} \mathrm{~F}\right)$. European Medicines Agency website. http://www.ema.europa.eu/docs/en_GB/document_library/EPAR__Product_Information/human/002422/WC500137635.pdf. Published 2012. Updated January 2013. Accessed July 8, 2015.

6. Vizamyl (flutemetamol F 18 injection). GE Healthcare website. http://www3. gehealthcare.com/en/Products/Categories/Nuclear_Imaging_Agents/Vizamyl. Published May 15, 2015. Accessed July 8, 2015.

7. EPAR summary for the public: Vizamyl. European Medicines Agency website. http://www.ema.europa.eu/docs/en_GB/document_library/EPAR_-_Product_ Information/human/002557/WC500172950.pdf. Accessed July 8, 2015. 
8. NEURACEQ (florbetaben F 18 injection), for intravenous use. http://neuraceq. com/images/Neuraceq_PI_031814.pdf. NeuraCeq website. Published 2014. Revised April 2014. Accessed July 10, 2015.

9. EPAR summary for the public: Neuraceq. European Medicines Agency website. http://www.ema.europa.eu/ema/index.jsp?curl=pages/medicines/human/medicines/ 002553/human_med_001716.jsp\&mid=WC0b01ac058001d124. Published November 3, 2014. Accessed July 8, 2015.

10. Rowe CC, Villemagne VL. Brain amyloid imaging. J Nucl Med Technol. 2013;41:11-18.

11. Sultana J, Cutroneo P, Trifiro G. Clinical and economic burden of adverse drug reactions. J Pharmacol Pharmacother. 2013:4(suppl 1):S73-S77.

12. Wong DF, Rosenberg PB, Zhou Y, et al. In vivo imaging of amyloid deposition in Alzheimer disease using the radioligand ${ }^{18} \mathrm{~F}-\mathrm{AV}-45$ (florbetapir F 18). J Nucl Med. 2010;51:913-920.

13. Mettler FA, Huda W, Yoshizumi TT, Mahesh M. Effective doses in radiology and diagnostic nuclear medicine: a catalog. Radiology. 2008;248:254-263.

14. Goerres GW, Hany TF, Kamel E, et al. Head and neck imaging with PET and PET/CT: artefacts from dental metallic implants. Eur J Nucl Med Mol Imaging. 2002;29:367-370.

15. Sureshbabu W, Mawlawi O. PET/CT imaging artifacts. J Nucl Med Technol. 2005;33:156-161.

16. Popilock R, Sandrasagaren K, Harris L, Kaser K. CT artifact recognition for the nuclear technologist. J Nucl Med Technol. 2008;36:79-81.

17. Choi SR, Golding G, Zhuang Z, et al. Preclinical properties of ${ }^{18} \mathrm{~F}-\mathrm{AV}-45$ : a PET agent for A $\beta$ plaques in the brain. J Nucl Med. 2009;50:1887-1894.

18. Snellman A, Rokka J, Lopez-Picon FR, et al. Pharmacokinetics of ${ }^{18} \mathrm{~F}$ flutemetamol in wild-type rodents and its binding to beta amyloid deposits in a mouse model of Alzheimer's disease. Eur J Nucl Med Mol Imaging. 2012;39:1784-1795.

19. Lee Y-S. Radiopharmaceuticals for molecular imaging. Open Nucl Med J. 2010;2:178-185.

20. AvSpeak: a glossary of aviation terms and abbreviations. Aerofiles website. http://www.aerofiles.com/glossary.html. Published June 30, 2008. Accessed July $10,2015$.

21. Keller SH, Sibomana M, Olesen OV, et al. Methods for motion correction evaluation using F-18 FDG human brain scans on a high-resolution PET scanner. J Nucl Med. 2012;53:495-504.

22. Ikari Y, Nishio T, Makishi Y, et al. Head motion evaluation and correction for PET scans with F-18 FDG in the Japanese Alzheimer's disease neuroimaging initiative (J-ADNI) multi-center study. Ann Nucl Med. 2012;26:535-544.

23. Green MV, Seidel Y, Stein S, et al. Head movement in normal subjects during simulated PET brain imaging with and without head restraint. J Nucl Med. 1994;35:1538-1546.

24. Beyer T, Tellmann L, Nickel I, Pietrzyk U. On the use of positioning aids to reduce misregistration in the head and neck in whole-body PET/CT studies. J Nucl Med. 2005;46:596-602.

25. Beyer T, Antoch J, Müller S, et al. Acquisition protocol considerations for combined PET/CT imaging. J Nucl Med. 2004;45(suppl):25S-35S.

26. ADNI-GO PET technical procedures manual: FDG \& AV-45. Alzheimer's Disease Neuroimaging Initiative website. https://adni.loni.usc.edu/wp-content/uploads/2010/ 05/ADNIGO_PET_Tech_Manual_01142011.pdf. Published January 14, 2011. Accessed July 10, 2015

27. Cherry SR, Sorenson JA, Phelps ME. Physics in Nuclear Medicine. 4th ed. Philadelphia, PA: Elsevier; 2012:307-343. 